\title{
Microcontroller Power Consumption Measurement Based on PSoC
}

\author{
Strahinja P. Janković, Student Member, IEEE, Vujo R. Drndarević, Member, IEEE
}

\begin{abstract}
Microcontrollers are often used as central processing elements in embedded systems. Because of different sleep and performance modes that microcontrollers support, their power consumption may have a high dynamic range, over $100 \mathrm{~dB}$. In this paper, a data acquisition (DAQ) system for measuring and analyzing the power consumption of microcontrollers is presented. DAQ system consists of a current measurement circuit using potentiostat technique, a DAQ device based on system on chip PSoC 5LP and Python PC program for the analysis, storage and visualization of measured data. Both Successive Approximation Register (SAR) and Delta-Sigma (DS) ADCs contained in the PSoC 5LP are used for measuring voltage drop across the shunt resistor. SAR ADC samples data at a 10 times higher rate than DS ADC, so the input range of DS ADC can be adjusted based on data measured by SAR ADC, thus enabling the extension of current measuring range by $\mathbf{2 8 \%}$. Implemented DAQ device is connected with a computer through a USB port and tested with developed Python PC program.
\end{abstract}

Keywords - DAQ, Microcontrollers, Power consumption, PSoC.

\section{INTRODUCTION}

$\mathrm{P}$ OWER management is an actual topic for both stationary and mobile electronic systems [1], [2]. In stationary systems it can be used to reduce generated heat and environmental impact as well as to reduce the electricity bills. In addition, the increased power dissipation increases the probability of device failure, and leads to an increase in prices due to the introduction of a system for cooling and packaging. In a wide range of portable electronic devices for computing, communication, biomedical and many other applications, power consumption represents an extremely important issue. Reducing power consumption when devices are battery powered allows the use of light batteries and longer device operation between the charging cycles. Many design methodologies are implemented to achieve an

Paper received May 15, 2016; revised June 27, 2016; accepted June 28, 2016. Date of publication July 20, 2016. The associate editor coordinating the review of this manuscript and approving it for publication was Prof. Branimir Reljin.

This paper is a revised and expanded version of the paper presented at the 23rd Telecommunications Forum TELFOR 2015 [30].

This work was supported by the Serbian Ministry of Education, Science and Technological Development through the project TR32043.

Corresponding Author, Strahinja P. Janković is Ph.D. candidate at the School of Electrical Engineering, University of Belgrade, Bul. kralja Aleksandra 73, 11120 Belgrade, Serbia (e-mail: jankovics@etf.bg.ac.rs).

Vujo R. Drndarević is with the School of Electrical Engineering, University of Belgrade, Bul. kralja Aleksandra 73, 11120 Belgrade, Serbia. energy-efficient electronic system [3]-[7]. For power management to be effective, the power consumption of power managed system needs to be accurately measured [8].

Power consumption of an embedded system depends on operating voltage and current. In order to measure and visualize voltage and current in real-time, oscilloscopes and data acquisition (DAQ) systems can be used. Oscilloscopes have high precision and allow real-time visualization and analysis of measured data. DAQ systems consist of sensor, DAQ device connected to a computer and PC software for data acquisition, visualization and analysis running on that computer [9].

When measuring power consumption of an embedded system, voltage is kept at a certain operating value (for the system to be functional) and current consumption is measured. If current is sampled at predefined time intervals, average energy and power consumption data can be obtained.

In this paper a new DAQ system for measuring microcontroller power consumption is presented.

This paper is organized in the following way: the section Measurement principles provides the concepts and details of measuring current with a high dynamic range, since microcontroller current consumption exhibits similar behavior. The section System implementation focuses on presenting three integral parts of the proposed solution: Programmable System on Chip (PSoC 5LP), Data acquisition device and Python PC program. Experimental results are presented in section Results and Discussion. Finally, conclusions are given in the last section.

\section{MEASUREMENT PRINCIPLES}

Current measurement using shunt is a widely used technique [10]. However, problem with shunt is the burden voltage effect [11], which affects operating voltage of the system whose power consumption is being measured. That is especially noticeable if current rapidly changes in time as a result of system activity and if the dynamic range of changes is several orders of magnitude.

Several shunt-based current measurement techniques exist which solve the problem with burden voltage [12][14]. One solution to the problem of burden voltage is to use current mirror (Fig. 1a).

As can be seen from Fig. 1a, current flowing through a system whose power consumption is measured is mirrored and shunt is placed in the mirrored branch. Thus the operating voltage remains constant, but accuracy of measurement is affected by the transistors and matching of those transistors. Also, power consumption of the whole 
system is increased and calibration is required.

Another possibility is to use the potentiostat technique [13], [14]. For providing a constant voltage supply for the device under test (DUT), operational amplifier with negative feedback is used. In this case, an OP amp with high input impedance, high slew rate and with the applicationappropriate maximum output current needs to be used. There are two variants of potentiostat technique, depending on the current sensing being low-side (Fig. 1b) or high-side (Fig. 1c). Low-side current sensing variant provides low common mode voltage on shunt resistor terminals, but requires a negative supply voltage to be available and disturbs ground path. High-side current sensing allows a supply voltage of DUT to be set using $V_{D U T}$, but has a high common mode voltage on shunt resistor terminals.

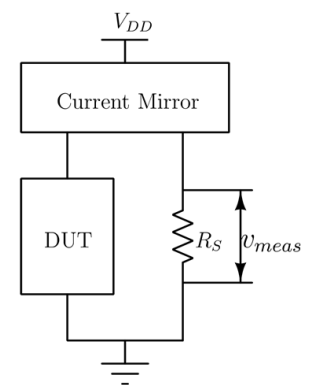

(a)

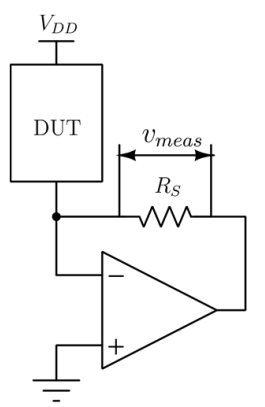

(b)

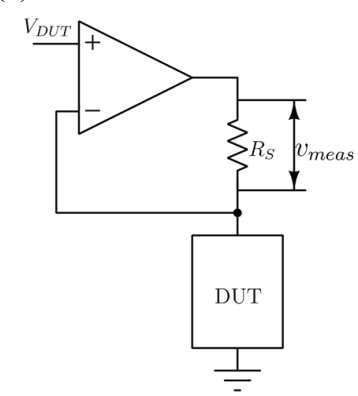

(c)
Fig. 1. Current measurement without burden voltage effect: a) current mirror; b) low-side potentiostat; c) high-side potentiostat.

Microcontrollers are widely used in embedded system products and a number of low-power devices or devices that are battery powered, such as industrial devices, consumer electronic devices, smart sensors and others. Battery life time is a critical parameter of these applications, in which the microcontroller has a very important role in reducing system power consumption and prolonging battery life.

Modern microcontrollers have many different peripherals integrated and support several active and sleep states, which are represented with different levels of functionality and power consumption. Also, frequency and voltage scaling, as well as clock gating are power management techniques widely used in microcontroller power consumption reduction [15], [16]. Because of different low power and performance modes and different power management techniques that microcontrollers support, current consumption of microcontrollers spans in the range of $10^{-6}$ to $10^{-1} \mathrm{~A}$ [17]. This high dynamic range of current values makes plain shunt technique inadequate for microcontroller current measurement, because with a single shunt resistor value measurements with the required accuracy over the entire range cannot be obtained. If shunt resistor value is small, measurement accuracy for low current will not be enough. On the other hand, if shunt resistor value is large, system functionality will be compromised when current consumption is high, because of the burden voltage effect.

There are several commercial solutions available for measuring power consumption of embedded systems: EnergyTrace [18], PowerMonitor [19], PowerScale [20]. An overview of their features is presented in Table 1, and they are discussed below.

TABLE 1: BASIC CHARACTERISTICS OF COMMERCIAL SOLUTIONS FOR POWER CONSUMPTION MEASUREMENT.

\begin{tabular}{lrr}
\hline Solution & $\begin{array}{r}\text { Current } \\
\text { range }\end{array}$ & $\begin{array}{r}\text { Maximum } \\
\text { sampling rate }\end{array}$ \\
\hline EnergyTrace & $\mathrm{nA}-\mathrm{mA}$ & $4.2 \mathrm{ksps}$ \\
PowerMonitor & $2 \mu \mathrm{A}-1 \mathrm{~A}$ & $5 \mathrm{ksps}$ \\
PowerScale & $0.2 \mu \mathrm{A}-1 \mathrm{~A}$ & $100 \mathrm{ksps}$ \\
\hline
\end{tabular}

EnergyTrace is used for power consumption measurement only for MSP430 microcontrollers and it uses the time density of the dc-dc controller charge pulses to measure energy consumption of microcontroller [18]. PowerMonitor and PowerScale use shunt for current measurement. PowerScale covers the widest current range, but it requires manual modification of measurement system for a full supported range.

\section{SYSTEM IMPLEMENTATION}

In this paper a new data acquisition system for measuring microcontroller power consumption is presented. DAQ device is based on PSoC 5LP system on chip [21], connected with a computer through a USB port. For the analysis, storage and visualization of measured data Python PC application is created.

The DAQ system should meet the following main requirements:

- current measurement from $1 \mu \mathrm{A}$ to $100 \mathrm{~mA}$,

- acquisition rate up to $10 \mathrm{ksps}$,

- external synchronization of events,

- software reconfiguration of the system suiting other applications

\section{A. Programmable System on Chip PSoC5 LP}

PSoC is a versatile Programmable System on Chip that, in addition to a powerful microcontroller, contains Programmable Logic Devices (PLD), circuits for analog signal processing and circuits for signal conversion. For the DAQ device design PSoC 5LP chip is used and its main features will be presented shortly [21].

PSoC 5LP is a family of system on chip from Cypress. It integrates Cortex-M3 microprocessor core with a range of configurable analog and digital blocks. Analog and digital blocks can be configured at design time using PSoC Creator IDE [22], and can have various functions: $\mathrm{AD}$ and DA converters, amplifiers, analog multiplexers, PWM components, serial interface, etc. A description of some 
functional blocks will be presented.

Successive Approximation Register (SAR) ADC supports 8 to 12 bit resolution and a sampling rate of up to 1 Msps. It can be used in single-ended and differential configuration. Internal $1.024 \mathrm{~V}$ reference can be used, as well as external.

Delta-Sigma (DS) ADC with selectable resolution from 8 to 20 bits and a sampling rate of up to $384 \mathrm{ksps}$ enables measurements in the microvolt range. It can be used in a single-ended or differential configuration. Internal $1.024 \mathrm{~V}$ reference can be used, as well as external. Additionally, input voltage range can be configured at design time. Supported voltage ranges for differential configuration are: $\pm V_{\text {ref }}, \pm V_{\text {ref }} / 2, \pm V_{\text {ref }} / 4, \pm V_{\text {ref }} / 8$ and $\pm V_{\text {ref }} / 16$, where $V_{\text {ref }}$ is a reference voltage used. Relations between resolution and maximum sampling rate for $\pm V_{\text {ref }}$ and $\pm V_{\text {ref }} / 16$ input ranges at $V_{\text {ref }}=1.024 \mathrm{~V}$, as two extremes, are given in Table 2 .

TABLE 2: MAXIMUM SAMPLING RATE FOR DS ADC FOR DIFFERENT RESOLUTION AND REFERENCE VOLTAGE.

\begin{tabular}{crr}
\hline $\begin{array}{l}\text { Input range } \\
\left(\boldsymbol{V}_{\text {ref }}=\mathbf{1 . 0 2 4} \boldsymbol{V}\right)\end{array}$ & $\begin{array}{r}\text { Resolution } \\
\text { [bits] }\end{array}$ & $\begin{array}{r}\text { Max sampling } \\
\text { rate [sps] }\end{array}$ \\
\hline \multirow{2}{*}{ $\pm V_{\text {ref }}$} & 15 & 113777 \\
& 16 & 48000 \\
& 17 & 12000 \\
& 18 & 3000 \\
\hline$V_{\text {reft }} 16$ & 15 & 27777 \\
& 16 & 15625 \\
& 17 & 3906 \\
& 18 & 976 \\
\hline
\end{tabular}

DS ADC has a differential amplifier front-end. Input amplifier provides high impedance input and can be used to amplify input signal with a maximum gain of 8 . DS ADC also supports up to 4 different independent configurations which can be configured through PSoC Creator IDE during software design and selected at runtime. For selected voltage range $V_{r}$, resolution $n$ of DS ADC and resistor $R_{S}$, minimum and maximum current values that can be measured are respectively:

$$
\begin{gathered}
I_{\min }=\frac{V_{r}}{2^{n-1} R_{S}}, \\
I_{\max }=\frac{V_{r}}{R_{S}}\left(1-\frac{1}{2^{n-1}}\right) .
\end{gathered}
$$

Term $2^{n-1}$ is used because only half of the available range is used, since measured voltage drop on shunt resistor will always be positive.

DMA controller supports copying data from memory to memory, memory to peripheral, peripheral to memory and peripheral to peripheral. Single transfer can be used for data up to 32 bit wide. There are 128 Transaction Descriptors which can be linked, so DMA requests can be cascaded.

$D A C$ supports 8 to 12 bit resolution and can provide output voltage up to $4.096 \mathrm{~V}$. Output voltage can be set at runtime.

USB Full speed controller is built into the chip. It supports all four data transfer types: Control, Interrupt, Bulk and Isochronous. USB controller can be configured at design time for a desired mode of operation.

\section{B. Data acquisition device}

DAQ device measures voltage drop across the shunt resistor and sends measured data, together with external synchronization data to PC through USB port. A block diagram of implemented DAQ device with measurement circuit is presented in Fig. 2.

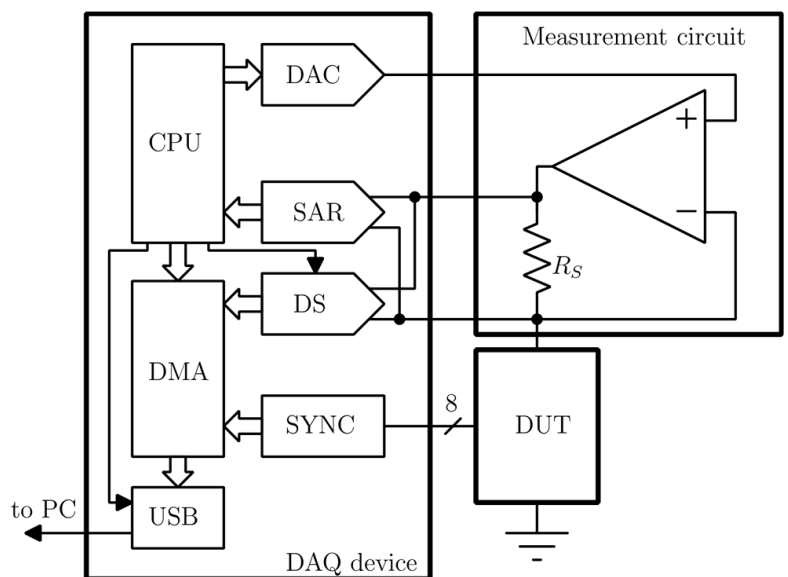

Fig. 2. DAQ device and measurement circuit block diagram.

DAC is used to set operating voltage for the device under test (DUT). DS ADC, because of its high resolution, is used for measuring voltage drop on shunt resistor. Internal 1.024 $\mathrm{V}$ reference is used $\left(V_{r e f}=1.024 \mathrm{~V}\right)$. Since $10 \mathrm{ksps}$ is a target acquisition rate, maximum resolution for DS ADC is 16 bits (Table 2). However, since common mode voltage is over 2 $\mathrm{V}$, input ranges of $\pm V_{\text {ref }} / 4, \pm V_{\text {ref }} / 8$ and $\pm V_{\text {ref }} / 16$ cannot be used with resolution higher than 15 bit because input capacitors will not be fully charged [23]. That is why resolution of 15 bits is used.

From equation (1), with DS ADC 15 bit resolution and voltage range equal to $\pm V_{\text {ref, a minimal value of shunt }}$ resistor is $62.5 \Omega$. However, shunt resistor of $62.5 \Omega$ would cause voltage drop for maximum current of $100 \mathrm{~mA}$ to be $6.25 \mathrm{~V}$, which is much higher than ADC's input voltage range. On the other hand, in order to measure low currents with high precision, input voltage range should be reduced. If the input voltage range is set to $\pm V_{\text {ref }} / 16$, then the minimal value of shunt resistor will be $3.9 \Omega$, and in this case the maximum value of the current which can be measured is 16 $\mathrm{mA}$.

In order to avoid the above-mentioned problems and still achieve a desired data acquisition rate, SAR ADC is set to acquire data with 8 bit resolution at $100 \mathrm{ksps}$, which is 10 times higher than the DS ADC data acquisition rate. All four available configurations for DS ADC are set to use different input ranges. This way, by selecting DS ADC configuration based on data measured by SAR ADC, input range of DS ADC can be set at runtime. Taking into account that PSoC CPU clock speed is several orders of magnitude greater than the DS ADC sampling rate, switching time is less than one sampling interval, which can lead to loss of at most one sample.

DS ADC configurations with selected input ranges and thresholds for initiating configuration change are shown in Table 3. 


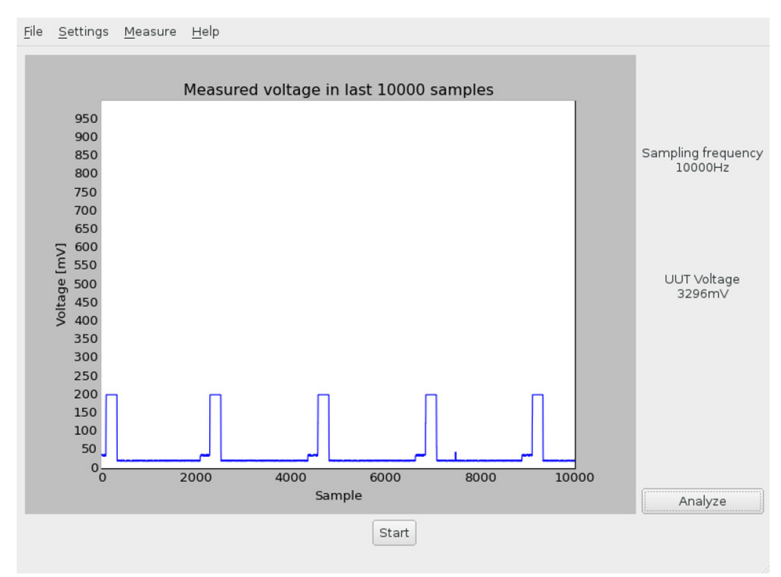

(a)

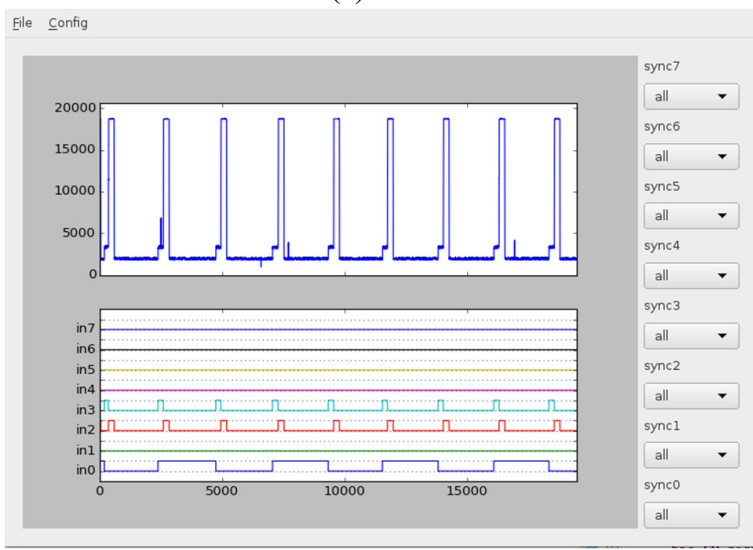

(c) "cycles":[ ], $\{$ "name":"period", "id":0 \} "states":[ \{ "name":"example", "ids":[ 2, 3 ], "state":[

\{ "name":"idle", "val":"0x0" \},

\{ "name":"adc", "val":"0x1" \}, ]

\{ "name":"transmit", "val":"0x2" \}

\}

]

\}

(e)

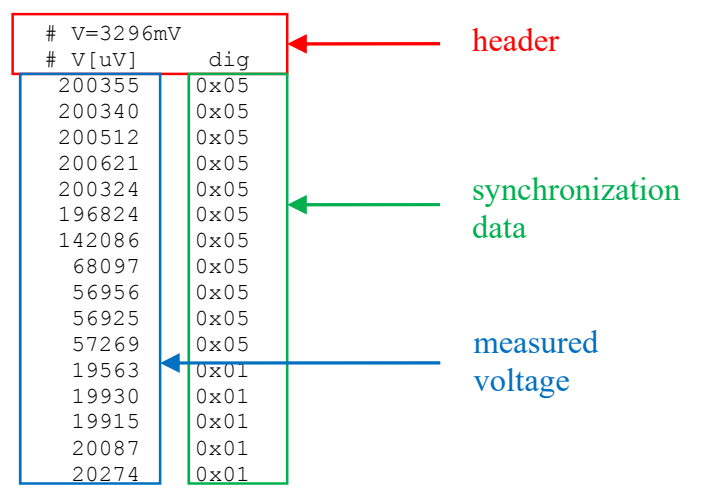

(b)

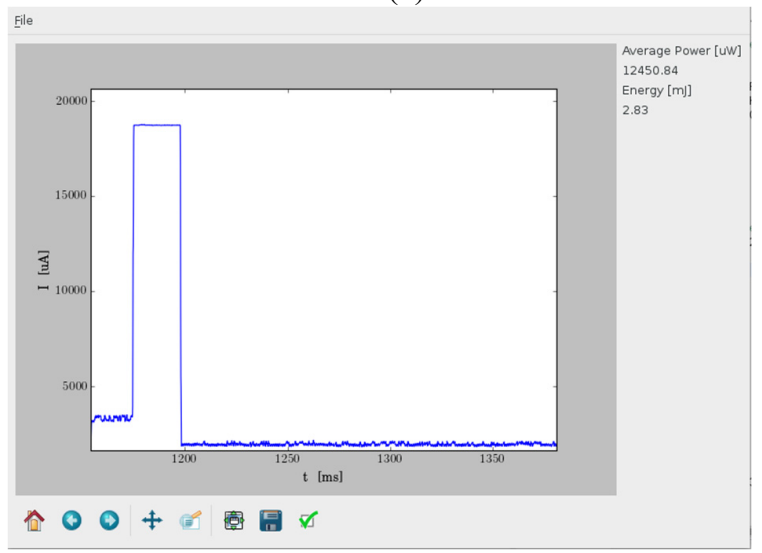

(d)

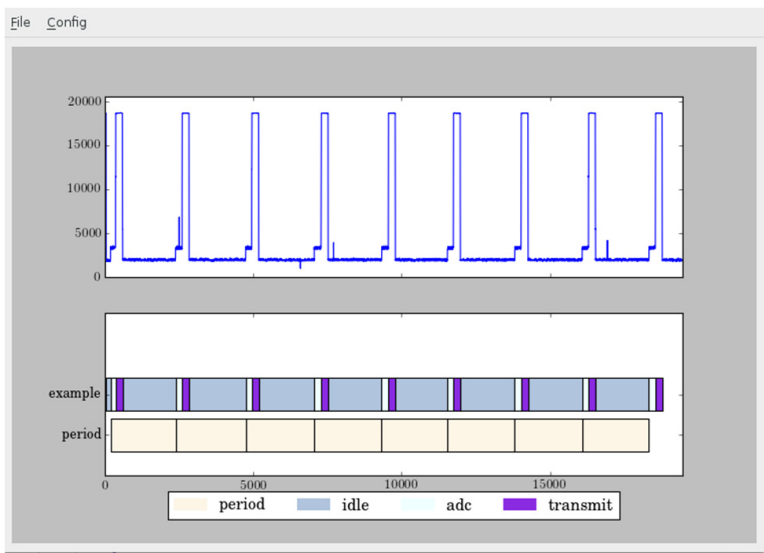

(f)

Fig. 3. PC Application: a) Real-time visualization during data acquisition; b) Format of stored data; c) Analysis window; d) Selected segment analysis and display; e) JSON file format for configuration of synchronization data interpretation;

f) Analysis window with synchronization data interpretation.

TABLE 3: DS ADC CONFIGURATIONS SETUP.

\begin{tabular}{llrr}
\hline Config & Input range & $V_{T H-}$ & $V_{T H+}$ \\
\hline 1 & $\pm 1.024 \mathrm{~V}$ & $218 \mathrm{mV}$ & $\mathrm{X}$ \\
2 & $\pm 0.256 \mathrm{~V}$ & $97 \mathrm{mV}$ & $242 \mathrm{mV}$ \\
3 & $\pm 0.128 \mathrm{~V}$ & $40 \mathrm{mV}$ & $113 \mathrm{mV}$ \\
4 & $\pm 0.064 \mathrm{~V}$ & $\mathrm{x}$ & $56 \mathrm{mV}$ \\
\hline
\end{tabular}

In order to meet timing requirements for data transfer, DMA is used. Measured voltage data is packed, together with information about DS ADC range and 8 digital channels value, and triple buffered. Each buffer can store up to 512 words with a word size of 32 bits. USB controller is configured for bulk transfer with 64 Bytes packet size. USB data transfer is managed by DMA.

Power consumption measurement is done using highside potentiostat technique (Fig. 1c). From equations (1) and (2) a minimum value of shunt resistor for the $V_{r}=0.064$ $\mathrm{V}$ is $3.9 \Omega$ and maximum is $10.24 \Omega$ which is achieved for $V_{r}=1.024 \mathrm{~V}$. Shunt resistor of $10 \Omega$ is chosen, which allows current measurement in the range from $0.39 \mu \mathrm{A}$ to 102.4 $\mathrm{mA}$. 


\section{PC program}

A PC program that provides data acquisition, analysis and visualization is implemented in Python. Python USB access module PyUSB [24] is used for communication with PSoC5 LP and module PyQt [25] for GUI. Matplotlib Python plotting package [26] is used for data graphs.

The main application window is presented in Fig. 3a. DAQ device parameters, DAC voltage and log file, can be set from Settings menu. After parameters are set, acquisition can be started and measured voltage drop on shunt resistor is visualized in real-time and stored.

Measurement results are stored in the format presented in Fig. 3b, so they can be used for further analysis. Header holds a supply voltage that was set for DUT using DAC. Data is organized so that the first column holds measured voltage in microvolts, and the second column holds 8 bit digital value from synchronization signals.

In the analysis window (Fig. 3c), data stored during acquisition can be displayed. Shunt resistor value can be set for current calculation. Measured current and synchronization signals are plotted and a segment for further analysis can be selected. Segment selection can be made based on synchronization signals, or an arbitrary section of data can be selected. A segment selected in analysis window is shown in a new window (Fig. 3d), together with calculated power and energy consumption.

User can define how synchronization signals will be interpreted by the analysis application. Synchronization bits can be interpreted either as cycle information, where every time synchronization bit is toggled, a new cycle starts, or as state information, where a group of synchronization bits contains information about a current state. Configuration can be passed as a JavaScript Object Notation (JSON) file in the format presented in Fig. 3e. Based on configuration and measured synchronization data timing diagram with information about cycles and state changes can be displayed (Fig. 3f). Clicking on a certain cycle or state will select a segment for further analysis (Fig. 3d).

\section{RESULTS AND DISCUSSION}

Presented data acquisition system for measuring and analyzing power consumption of microcontrollers has been tested by measuring the power consumption of CC430F5137 microcontroller [27]. Microcontroller CC430F5137 has MSP430 CPU core with CC1101 wireless interface. Microcontroller CC430F5137 was chosen because power consumption in different operating modes and with RF interface turned on or off differs significantly (Table 4).

TABLE 4: CURRENT CONSUMPTION OF MICROCONTROLLER CC430F5137 IN DIFFERENT CONFIGURATIONS

\begin{tabular}{lr}
\hline Configuration & $\boldsymbol{I}[\boldsymbol{m} \boldsymbol{A}] \boldsymbol{a 3 . 0 V}$ \\
\hline LPM3, RF off & 0.001 \\
LPM0, RF off & 0.08 \\
Active, RF off & $0.23-5$ \\
Active, RF on, RX & 15 \\
Active, RF on, TX & 18 \\
\hline
\end{tabular}

CC430F5137 microcontroller is programmed to perform the following repetitive task: acquire 256 samples from AD converter, calculate the average of acquired data and send acquired samples over RF link. One digital pin was set to toggle every time a new cycle starts. Two additional pins were used to indicate ADC averaging, Transmit and Idle states.

Results of microcontroller power consumption measurement and analysis during one cycle of example program are shown in Fig. 3d. The total energy consumption of one cycle is $2.83 \mathrm{~mJ}$, where energy consumed in ADC averaging state is $0.22 \mathrm{~mJ}$, Transmit state $1.44 \mathrm{~mJ}$ and Idle state $1.17 \mathrm{~mJ}$.

The performance and characteristics of DAQ system presented in this paper are comparable with current commercial power consumption measurement systems, especially in the range of interest for microcontroller power consumption. The advantage of presented measurement system in relation to what is commercially available is that it requires no manual adjustment at runtime. By using potentiostat measuring technique, operating voltage of the system whose power consumption is measured is held constant, so there is no burden voltage effect.

\section{CONCLUSION}

In this paper the architecture, implementation and testing results for microcontroller power consumption measurement system are presented. Power consumption is measured by keeping constant DUT voltage supply $V_{D U T}$ and measuring current that is consumed. Supported current consumption measurement range is $0.39 \mu \mathrm{A}$ to $102.4 \mathrm{~mA}$ with a maximum sampling rate of $10 \mathrm{ksps}$. Up to eight external synchronization signals can be used to provide information for interpretation of measured data. This high dynamic range for current measurement is achieved by using both types of ADCs present in PSoC 5LP so DeltaSigma ADC's input range is modified at runtime based on data sampled by Successive Approximation Register ADC, which uses a 10 times higher acquisition rate. The advantage of presented DAQ device over commercially available ones is that there is no burden voltage effect and it requires no manual adjustment at runtime. PC application program for real-time data visualization and storage, and offline analysis and interpretation of measured data is implemented in Python.

It should be noted that the proposed solution uses an external operational amplifier for the implementation of potentiostat. DAQ device can be modified to use only components inside PSoC 5LP, but this would limit the measurement range to $25 \mathrm{~mA}$ [28]. With a different shunt resistor value or modification to the potentiostat range DAQ device can be adapted to measure the power consumption of different embedded systems.

A limitation of proposed system is that it can be used to measure the power consumption of only those embedded systems whose voltage supply is less than $5 \mathrm{~V}$, because of the maximum input voltage on PSoC pins [29]. This can be solved by using an external differential amplifier, whose output would be used as input to DAQ device ADCs. 


\section{REFERENCES}

[1] Y. Baruskov, "Battery Power Management for Portable Devices", Artech House, 2013

[2] Y. Son, T. Pulkkinen, K. Moon, "Home energy management system based on power line communication", IEEE Transactions on Consumer Electronics Vol. 56, Issue 3, pp. 1380-1386, 2010.

[3] L. Benini, G. DeMicheli, "Dynamic Power Management: Design Techniques and CAD Tools", Springer, 1998.

[4] T. D. Burd, R. W. Brodersen, "Energy Efficient Microprocessor Design", Springer, 2012.

[5] K. Gorman, A. Cusson, A. Lukomski, "Real-time energy efficient embedded system development methodology", IEEE Digital Signal Processing and Signal Processing Education Meeting (DSP/SPE), 2013, pp. 284-289.

[6] F. B. Muslim, A. Qamar, L. Lavango, "Low power methodology for an ASIC design flow based on high-level synthesis", 23rd International Conference on Software, Telecommunications and Computer Networks (SoftCOM), 16-18 Sept. 2015., pp. 11-15.

[7] J. M. Rabaey, M. Pedram, "Low Power Design Methodologies", Springer, 1996.

[8] D. Hackenberg, T. Ilsche, R. Schone, D. Molka, M. Schmidt, W.E. Nagel, "Power measurement techniques on standard compute nodes: A quantitative comparison". International Symposium on Performance Analysis of Systems and Software (ISPASS) (2013), pp. 194-204.

[9] "What is Data Acquisition: Parts of DAQ system", National Instruments Corporation, 2016. [Online]. Available: http://www.ni.com/data-acquisition/what-is/

[10] S. Ziegler, R. C. Woodward, IU, H.H.C. \& L. J. Borle (2009). Current Sensing Techniques: A Review. IEEE SENSORS JOURNAL, 9(4), 354-376.

[11] L. Schreier, "How to Minimize Errors for Low-Current Measurements", National Instruments, 2011. Available: http://www.ni.com/tutorial/5448/en/

[12] Z. Nakutis, "Embedded Systems Power Management Methods Overview," Measurements, vol. 2, no. 44, pp. 29-35, 2009.

[13] R. Greef, "Instruments for use in electrode process research," Journal of Physics E: Scientific Instruments, vol. 11, no. 1, pp. 1-12, Jan. 1978.

[14] A. Di Nisio, T. Di Noia, C. G. C. Carducci, and M. Spadavecchia, "High Dynamic Range Power Consumption Measurement in
Microcontroller-Based Applications," IEEE Transactions on Instrumentation and Measurement, pp. 1-9, 2016.

[15] A. M. Holberg and A. Saetr, "Innovative Techniques for Extremely Low Power Consumption with 8-bit Microcontrollers," ATMEL, White paper 7903A - AVR - 2006/02, 2006.

[16] I. T. Popovic and S. P. Jankovic, "Frequency scaling for low-power embedded system," in Telecommunications Forum (TELFOR), 2012 20th, 2012, pp. 1096-1099.

[17] "Using STM32F4 MCU power modes with best dynamic efficiency," ST, Application Note AN4365, May 2014.

[18] "Code Composer Studio v6.1 for MSP430 User Guide", Texas Instruments Inc., Dallas, TX, 2015, pp. 20-35.

[19] "Mobile Device Power Monitor Manual", Monsoon Solutions Inc., Bellevue, WA, 2014.

[20] "PowerScale brochure", Hitex GmbH, Karlsruhe, Germany, 2007.

[21] "PSoC 5LP Architecture Technical Reference Manual", Cypress Semiconductor, San Jose, CA, 2013.

[22] Cypress Semiconductor, "PSoC Creator Integrated Design Environment (IDE)", 2016. [Online]. Available: http://www.cypress.com/products/psoc-creator-integrated-designenvironment-ide

[23] "PSoC5LP DelSig problem with input range $+/$-Vref/4, /8, /16," in Cypress Developer Community Forum, 26. Feb 2015. [Online]. Available: http://www.cypress.com/comment/245911\#comment245911.

[24] W. L. Costa, "PyUSB 1.0", Available: https://walac.github.io/pyusb/

[25] B. M. Harwani. "Introduction to Python Programming and Developing GUI Applications with PyQT (1st ed.)". Course Technology Press, Boston, MA, United States, 2011.

[26] J. D. Hunter, "Matplotlib: A 2D graphics environment," Computing in science and engineering 9, no. 3 (2007): 90-95.

[27] "CC430 Family User's Guide", Texas Instruments Inc., Dallas, TX, 2013.

[28] "Operational Amplifier (Opamp)", PSoC Creator Component Datasheet, Cypress Semiconductor, San Jose, CA, 2015.

[29] "PSoC 5LP: CY8C58LP Family Datasheet", Cypress Semiconductor, San Jose, CA, 2015.

[30] S. P. Janković and V. R. Drndarević, "Microcontroller power consumption measurement based on $\mathrm{PSoC}$," Telecommunications Forum Telfor (TELFOR), 2015 23rd, Belgrade, 2015, pp. 673-676. 